\title{
Chromosomal characteristics of salt stress heritable gene expression in the rice genome
}

\author{
Matthew T. McGowan ${ }^{1 *}$, Zhiwu Zhang ${ }^{1,2}$ and Stephen P. Ficklin ${ }^{1,3}$
}

\begin{abstract}
Background: Gene expression is potentially an important heritable quantitative trait that mediates between genetic variation and higher-level complex phenotypes through time and condition-dependent regulatory interactions. Therefore, we sought to explore both the genomic and condition-specific characteristics of gene expression heritability within the context of chromosomal structure.
\end{abstract}

Results: Heritability was estimated for biological gene expression using a diverse, 84-line, Oryza sativa (rice) population under optimal and salt-stressed conditions. Overall, 5936 genes were found to have heritable expression regardless of condition and 1377 genes were found to have heritable expression only during salt stress. These genes with salt-specific heritable expression are enriched for functional terms associated with response to stimulus and transcription factor activity. Additionally, we discovered that highly and lowly expressed genes, and genes with heritable expression are distributed differently along the chromosomes in patterns that follow previously identified high-throughput chromosomal conformation capture ( $\mathrm{Hi}-\mathrm{C}) \mathrm{A} / \mathrm{B}$ chromatin compartments. Furthermore, multiple genomic hot-spots enriched for genes with salt-specific heritability were identified on chromosomes 1, 4, 6, and 8. These hotspots were found to contain genes functionally enriched for transcriptional regulation and overlaps with a previously identified major QTL for salt-tolerance in rice.

Conclusions: Investigating the heritability of traits, and in-particular gene expression traits, is important towards developing a basic understanding of how regulatory networks behave across a population. This work provides insights into spatial patterns of heritable gene expression at the chromosomal level.

Keywords: RNAseq, Genetics, Transcriptomics, Heritability, Agronomy

\section{Background}

Understanding the molecular mechanisms by which genetic variation influences complex quantitative traits remains a major goal of genetic research today. Current polygenic and omnigenic models posit that for complex traits, only a small proportion of heritable phenotypic variation can be explained by relatively few easily identified mutations with large effects. The remaining majority

\footnotetext{
* Correspondence: matt.mcgowan@wsu.edu

${ }^{1}$ Molecular Plant Sciences Program, Washington State University, French Ad 324G, Pullman, WA 99164, USA

Full list of author information is available at the end of the article
}

of heritable variation is due to a much larger quantity of low to moderate effect mutations. After more than a decade of research utilizing Genome-Wide Association Studies (GWAS) it is clear that many of these low to moderate effect genetic variants underlying complex traits tend to lie in regulatory regions of the genome rather than in protein coding regions. Furthermore, affected regions have been found to be enriched for genes that interact in highly interconnected regulatory networks [1]. Therefore, expression quantitative trait locus (eQTL) studies seek to identify relationships between genetic variants and the genes on which they may have a

(C) The Author(s). 2021 Open Access This article is licensed under a Creative Commons Attribution 4.0 International License, which permits use, sharing, adaptation, distribution and reproduction in any medium or format, as long as you give appropriate credit to the original author(s) and the source, provide a link to the Creative Commons licence, and indicate if changes were made. The images or other third party material in this article are included in the article's Creative Commons licence, unless indicated otherwise in a credit line to the material. If material is not included in the article's Creative Commons licence and your intended use is not permitted by statutory regulation or exceeds the permitted use, you will need to obtain permission directly from the copyright holder. To view a copy of this licence, visit http://creativecommons.org/licenses/by/4.0/ The Creative Commons Public Domain Dedication waiver (http://creativecommons.org/publicdomain/zero/1.0/) applies to the data made available in this article, unless otherwise stated in a credit line to the data. 
regulatory effect by treating gene expression as the phenotypic trait for GWAS analysis.

The increasing number of studies investigating eQTLs in multiple plant species have revealed similar patterns of eQTL architectures. The location of eQTLs in relation to their affected gene are often referred to as cis and trans depending on whether they map respectively to the same relative location as the gene or elsewhere in the genome. While cis eQTLs tend to have larger effects on average compared to trans eQTLs, only a small proportion of genes appear to have cis eQTLs that explain a majority of their expression variance. Instead, many genes appear to have both cis and trans acting eQTLs with the most eQTLs being trans $[2,3]$. Cross-gene eQTL analysis has revealed that many of these trans eQTLs are significantly enriched in genomic hotspots with wide reaching effects on gene expression $[4,5]$.

In any association study (GWAS or eQTL) characterization of heritability for the selected trait (e.g. phenotype or expression-level) is necessary to estimate genetic causality for the trait. Heritability is a fundamental genetics concept that describes how much of the variation in a given trait can be attributed to genetic variation [6]. It has demonstrated lasting usefulness in quantifying response to selection in plant breeding [7] and estimating disease risk in medicine [8]. Traditionally, heritability is estimated using known information about the genetic relationships between individuals. In human research, these known genetic relationships are usually in the form of monozygotic (identical) and dizygotic (fraternal) twins. In plant and animal research, pedigrees from controlled breeding populations are used to represent these genetic relationships. Another approach for estimating heritability uses high-density genotyping technologies such as single nucleotide polymorphism (SNP) arrays to infer genetic relationships. Genotype differences between individuals are used to calculate a genetic relationship matrix (GRM), also called a kinship matrix. This GRM is then used to estimate the proportion of phenotypic variance explained using linear mixed models. This approach is referred to as Genomic Relatedness Restricted Maximum Likelihood (GREML) and has multiple software implementations such as GCTA [9], EMMA [10], and rrBLUP [11]. Despite the large number of eQTL studies investigating gene expression, relatively few studies have explored genomic patterns of gene expression heritability using GREML-based estimates. Two studies in humans explored gene expression heritability of whole blood samples [12, 13], but similar research in plants is currently lacking.

Another area of gene expression research that is relatively unexplored is the influence of environmental factors. Even though differential gene expression analysis is a highly active area of research, studies investigating variation in gene expression in response to environmental changes have primarily focused on condition, time, and tissue-specific expression variation. Yet these studies are limited to a few different genotypes, far below the necessary sample sizes required for performing eQTL analysis [14]. However, given that complex agronomic phenotypes are known to have significant genotype-byenvironment interaction effects, exploring how these interactions affect gene expression variation may provide novel insights into the underlying architecture of these phenotypes.

An important consideration prior to exploration of heritability is understanding any potential bias from variation that underlies the bimodal distribution of gene expression. It has been shown that gene expression when quantified with RNA-seq data has a bimodal structure such that lowly expressed (LE) genes and highly expressed (HE) genes appear as two overlapping distributions with LE genes centered in the negative $\log 2$ range and the other in the positive log2 range [15]. The source of this bimodality is a currently a topic of debate. One theory suggests the lower distribution is due to an unknown combination of transcriptional noise, ambiguous read mapping, contamination, cell type heterogeneity, and sequencing errors. Thus, many only use the HE genes for downstream research [16]. However, there is evidence that transcripts from the low abundance distribution are transcribed mRNA and not artifacts or small RNA molecules [17].

Another consideration for exploration of gene expression heritability, related to non-normal gene expression distributions, is that transcriptional repression has been shown to be correlated with the 3D conformational structure of chromosomes in the nucleus including chromatin and centromeric structures [18]. Chromatin alteration in plants has been shown to play important roles in tissue-specific specialization $[19,20]$, stress response [21-23], and suppression of transposable elements [24, 25]. Plant genomes have been found to possess active and repressive genome territories referred to as the $\mathrm{A}$ and $\mathrm{B}$ compartments which correspond to euchromatic and heterochromatic regions, respectively $[26,27]$. While these compartments have been found to be largely stable across tissues, it remains unclear how stable these compartments are across changing environmental conditions known to alter chromatin states such as abiotic stress.

In this study, we sought to address the limitations and considerations just described for gene expression heritability by exploring the 2D and 3D chromosomal characteristics of heritable gene expression using an RNA-seq dataset of 84 individuals of the Oryza sativa Rice Diversity Panel 1 (RDP1) previously reported [28]. We explored patterns of missing values in the RNA-seq data 
(i.e., missingness) and the distribution of highly expressed (HE) and lowly expressed (LE) genes across the 2D chromosomal structure. Heritability was calculated independently for salt stress and control conditions and their distribution was also explored across the 2D genomic structure. We then explored the relationship of $\mathrm{HE}$ and $\mathrm{LE}$ genes to the $\mathrm{Hi}-\mathrm{C}$ analysis of rice chromatin structures.

\section{Results}

\section{Gene expression}

For the 55,986 annotated gene transcripts in the Michigan State University (MSU) v7.0 Oryza sativa Nipponbare (rice) assembly [29], the distribution of missing values (genes with no measured expression) followed a U-shaped distribution with most genes having either a high or low missing rate and relatively few genes having moderate levels of missingness. We classified genes as having constitutive, mixed, or repressed expression patterns if non-zero expression was observed in $>95 \%$, 5 $95 \%$, or $<5 \%$ of samples, respectively (Fig. 1a). Overall, non-zero gene expression followed a clear bi-modal distribution consisting of a mode of HE genes with positive $\log _{2}$ TPMs and a second mode of LE genes with negative $\log _{2}$ TPMs (Fig. 1b). Genes with constitutive expression occupied the HE mode, while genes with a mixed or repressed expression pattern matched the LE mode. Thus, HE genes are both highly expressed and highly present (few missing values) while LE genes are lowly expressed and lowly present. Furthermore, cross-tabulation across conditions indicates that genes had largely conserved expression patterns for all three expression patterns (Table 1). While there were a small number of genes that switched categories between conditions, there were no genes that changed from constitutive to repressed.

\section{Heritability}

\section{Comparison of heritability results}

Correlation of gene expression biological replicates on a per-gene basis was calculated as a potential estimate for heritability, similar to twin-based measures of heritability in humans. Replicate heritability values were then compared to both GREML estimates of heritability using a genotypic mean (two-step) and GREML estimates that included replication as a random effect in the model.

Due to the relatively small sample size, there were many genes where the GREML heritability (single-step or two-step) could not be reliably predicted with a mixed linear model resulting in an inflated number of genes with low heritability estimates (0-0.2) and a wide 95\% confidence interval (Additional File 1, Fig. S1). There was strong correlation between replicate heritability versus single-step GREML ( $\rho=0.89)$, indicating that gene expression heritability can be estimated using the biological replicates expression data. However, the correlation of the two-step method was moderate when

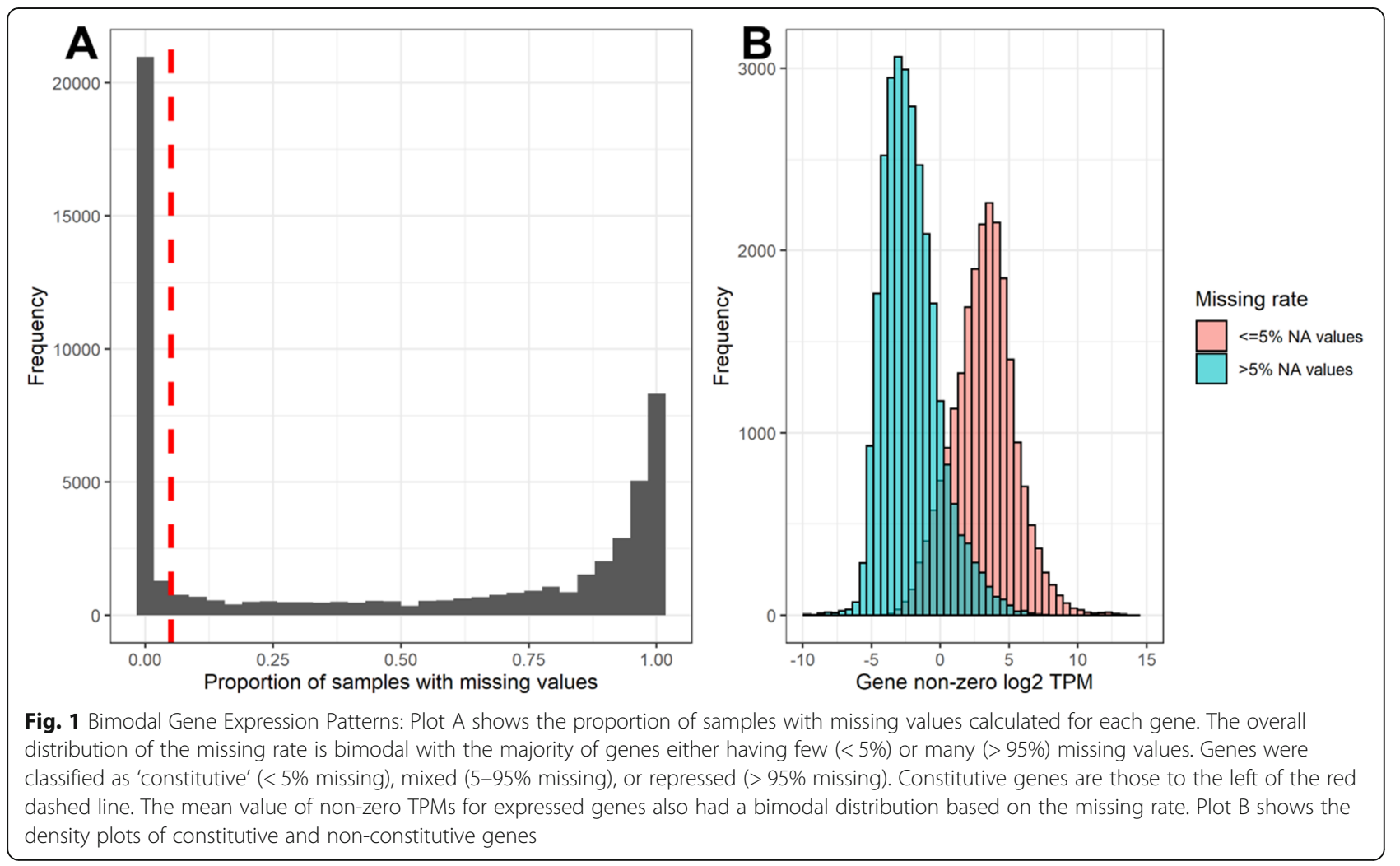


Table 1 Contingency Table of Expression-Level Categories

\begin{tabular}{llllll}
\hline & & \multicolumn{2}{l}{ Salt-stress } & & \\
\cline { 3 - 5 } & & Constitutive & Mixed & Repressed & Totals \\
\hline Control & Constitutive & 16,372 & 363 & 0 & 16,735 \\
& Mixed & 91 & 25,116 & 932 & 26,139 \\
& Repressed & 0 & 1007 & 12,105 & 13,112 \\
& Totals & 16,463 & 26,486 & 13,037 & \\
\hline
\end{tabular}

compared to the one-step approach $(\rho=0.41)$ and with replicate heritability approach $(\rho=0.45)$ (Fig. 2). Results in Fig. 2 are for the control condition, but patterns were similar for the salt condition (Additional File 1, Fig. S2).

\section{Condition-specific heritability classification}

To identify a significance threshold for expression heritability, randomized permutation tests of shuffled gene expression values were used to calculate a null heritability distribution. Using this null-distribution, a significance threshold was calculated using a fixed type-I error rate $(\square<=0.01$ ) (Fig. 3a). Genes were classified whether they were significantly heritable for control and saltstress conditions (Fig. 3b). While most genes with heritable expression appeared to have conserved heritability for both control and salt-stress conditions $(n=6851)$, there were a considerable number of genes significantly heritable only during control $(n=3599)$ or salt-stress $(n=1377)$. These genes with condition-specific heritability were less heritable than genes that were heritable across both conditions (Additional File 1, Fig. S3). Genes heritable in both salt stress and control were correlated symmetrically along the diagonal (Fig. 3b), indicating no condition-specific bias.

\section{Chromosomal structure and conformation \\ $H E$ and $L E$ genes follow distinct $2 D$ spatial patterns}

The spatial distribution of constitutive, mixed, and repressed genes was visualized along the chromosomes using a sliding window of $3 \mathrm{Mb}$ at $100 \mathrm{~Kb}$ intervals. Empirically, constitutive genes appear enriched on the ends

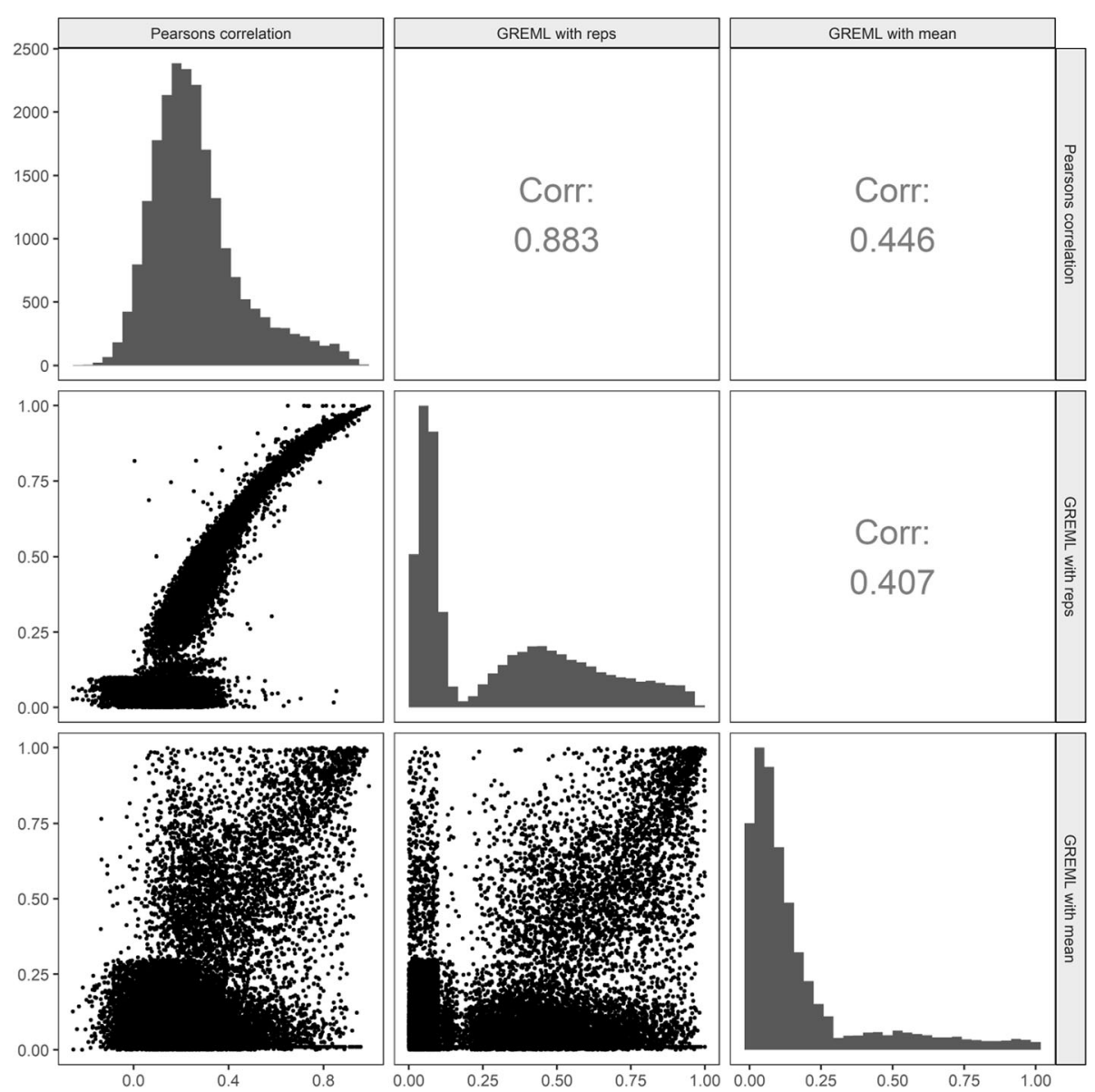

Fig. 2 Comparison of Heritability Calculation Methods for the Control condition: Pairwise correlation between repeatability (Pearson's), single-step GREML (with replicates), and two-step GREML (using the genotypic mean) for the control condition. The lower triangle shows correlation scatterplots of the pairwise comparisons, the diagonal provides the density distribution plots for each individual method and the upper right triangle provides the corresponding pairwise correlation values 

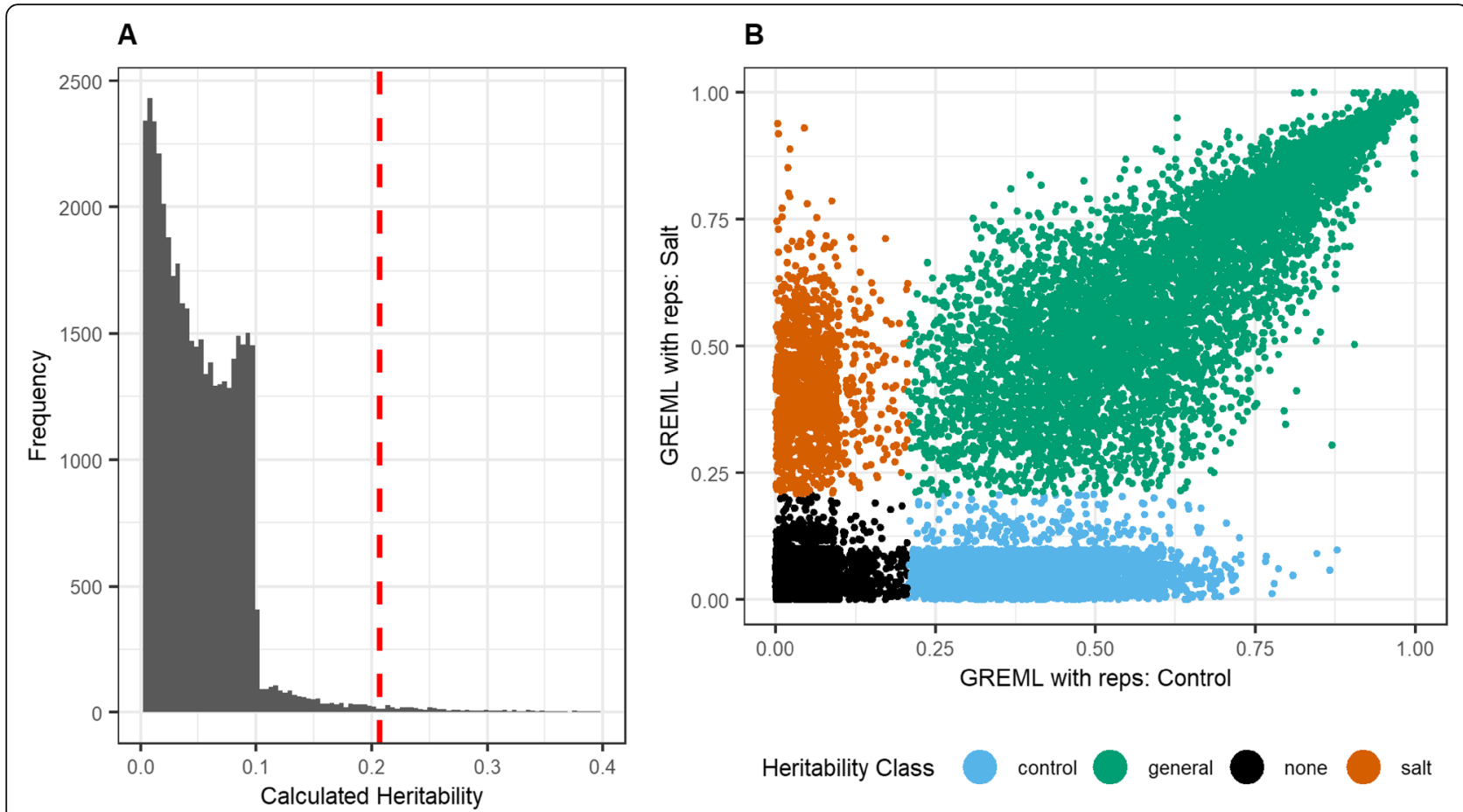

Fig. 3 Classification of gene expression heritability. Plot A shows the heritability distribution of randomly shuffled gene expression values. This distribution serves as the null-distribution used for determining non-significant heritability estimates for genes. The dashed red line indicates the quantile for a fixed type-1 error ( $\square=0.01$ ). Plot B shows the comparison of salt and control heritability estimates. A quantile threshold was used to classify each gene as having significant heritability in salt treatment, control or general (i.e. both)

of chromosomes and depleted near pericentromeric regions (Fig. 4). For metacentric chromosomes, this pattern formed a U-shape centered on the centromere. Densities for genes with repressed and mixed expression were often inverse of constitutive genes and appear enriched near the centromere and depleted at the chromosome ends. Reductions in density of constitutive genes were not always centered on the centromeric regions. For example, subtelocentric chromosomes 4, 9, and 10 (and chromosome 11 to a lesser extent) show this asymmetry as the short chromosomal arms appeared relatively devoid of genes with constitutive expression (Fig. 4).

\section{Comparison of gene expression and $\mathrm{HI}-\mathrm{C}$ a/B chromatin compartments}

Regarding 3D characteristics of expressed genes, densities of genes (when calculated using a fixed $100 \mathrm{~kb}$ window size) were highly correlated $(\rho=0.7-0.9)$ with $\mathrm{A} / \mathrm{B}$ chromatin compartments identified with the first principal component of PCA analysis of a $\mathrm{Hi}-\mathrm{C}$ contact map [27] (Additional File 1, Figs. S4-S6). Euchromatic A compartments corresponded to genes that were constitutively expressed across all genotypes. Conversely, heterochromatic B corresponded to genes with either mixed or repressed expression across genotypes.

\section{Salt-specific spatial enrichment analysis}

When the spatial distribution of genes with salt-specific heritability was compared to the distribution of genes with non-specific heritability, 22 windows were identified on chromosomes $1,4,6$, and 8 that passed a permutation-based $p$-value threshold ( $\square=0.001$ ) (Fig. 5, Table 2). This test indicates where the genome is enriched for salt-stress specific expression. Other chromosomes did not have significantly enriched windows (Additional File 1, Figs. S7-S9). Adjacent and overlapping windows were combined into five contiguous regions (Additional File 2, Table S1). Gene ontology enrichment analysis of heritable genes in these regions identified terms of transcription factor activity (GO: 0003700), response to endogenous stimulus (GO: 0009719), nucleic acid binding (GO:0003676), and DNA binding (GO:0003677) (Additional File 2, Tables 2-3). When compared to previous GWAS studies, there were overlaps between these regions and QTLs identified for salt-tolerance related traits. In particular, a $3 \mathrm{Mb}$ window on chromosome 4 directly overlaps with a highly significant $575 \mathrm{~Kb}$ QTL identified from a previous GWAS that used the same RDP1 panel that was significant for sodium and potassium accumulation in root tissue [28]. Fine mapping of this QTL identified HKT1;1, a sodiumtransporter gene (LOC_Os04g51820) that is the likely 

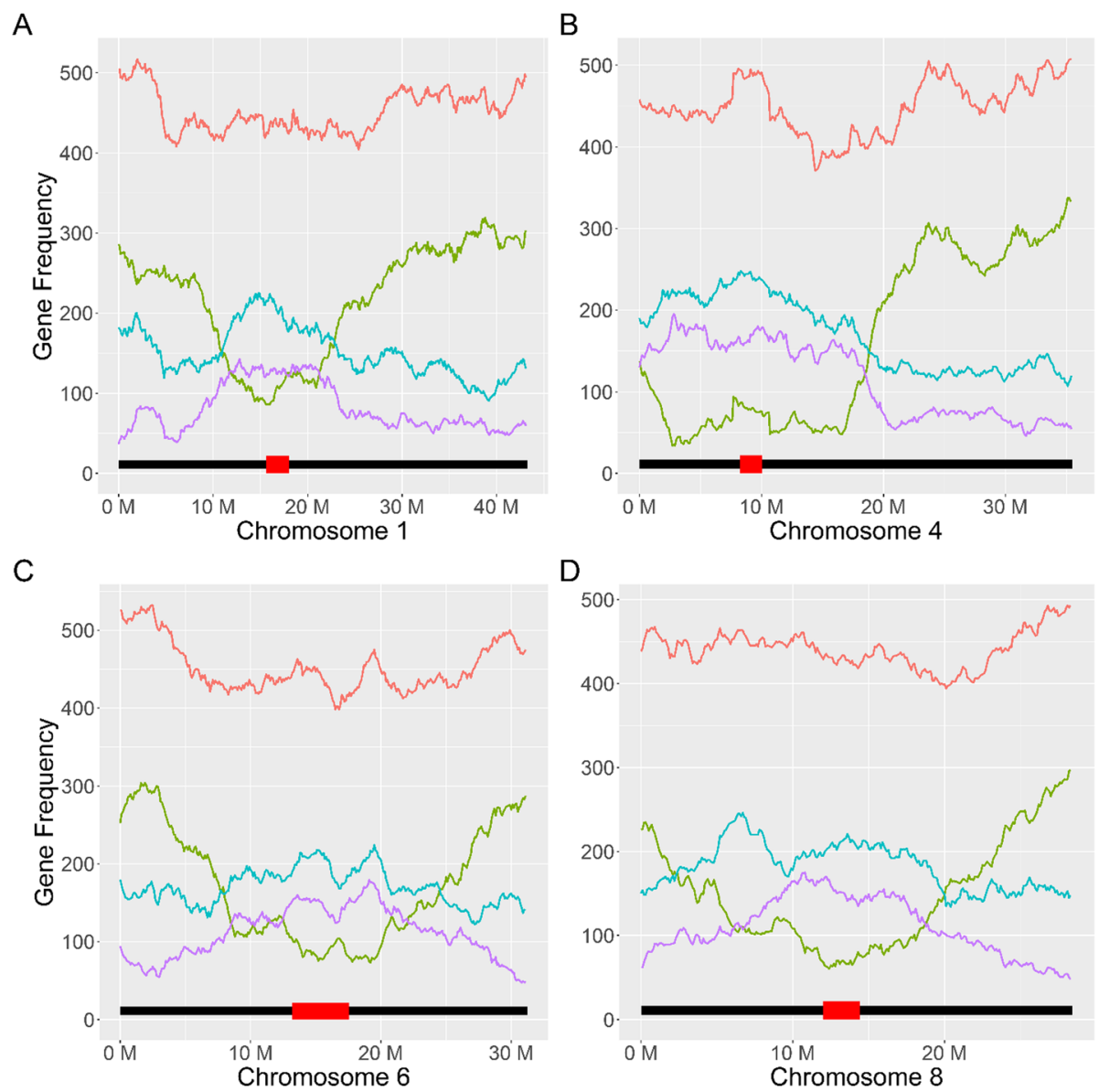

Fig. 4 Gene density distributions across chromosomes. Plots A-D represent chromosomes 1, 4, 6, and 8 respectively. The black lines at the bottom of each plot represent the relative chromosome length, with the position and relative size of pericentromeric regions indicated by overlapping red boxes. Overall gene frequency represented by the red line appears roughly uniform across each chromosome. Genes with constitutive expression (expressed in $>95 \%$ of samples), represented by the lime-colored line, are enriched on the distal ends of chromosome arms and depleted near pericentromeric regions. Genes with repressed expression ( $<5 \%$ of samples), represented by the cyan colored line, are enriched near pericentromeric regions. Genes with mixed expression (5-95\% of samples), represented by the pink line, largely follow the same distribution as repressed genes

causal gene. It was also determined that altering the expression of this gene using RNA-interference lines significantly affected both shoot and root growth under saline conditions [28].

In summary, results show missingness is the cause of bimodality in the salt-stress gene expression data. Regarding 2D characteristics, HE and LE genes have distinct distribution patterns in relation to the centromeric location of the chromosomes. Additionally, salt-specific heritable genes follow similar 2D distribution patterns but are also highly correlated with $3 \mathrm{D}$ conformation following $\mathrm{Hi}-\mathrm{C}$ identified $\mathrm{A} / \mathrm{B}$ compartments. We also identified several significant genomic hot-spots enriched for genes with salt-specific heritability on chromosomes 4 which is concordant with previous GWAS studies investigating salt tolerance phenotypes in a similar population as well as 3 additional windows on chromosomes 1,6 , and 8 .

\section{Discussion}

\section{Gene expression}

It has been suggested that low abundance mRNA identified in the LE distribution of TPM values may not be transcribed into proteins. Comparisons between lowly abundant genes in human metazoan cells and proteome quantification in human embryonic cells did not indicate that LE genes are translated [17]. While the results presented here do not definitively answer the question of whether LE genes are translated, the patterns observed both in the bimodal distribution (Fig. 1) and the crossconditional table (Table 1) provide insight regarding variation of transcriptional repression. Genes with few 

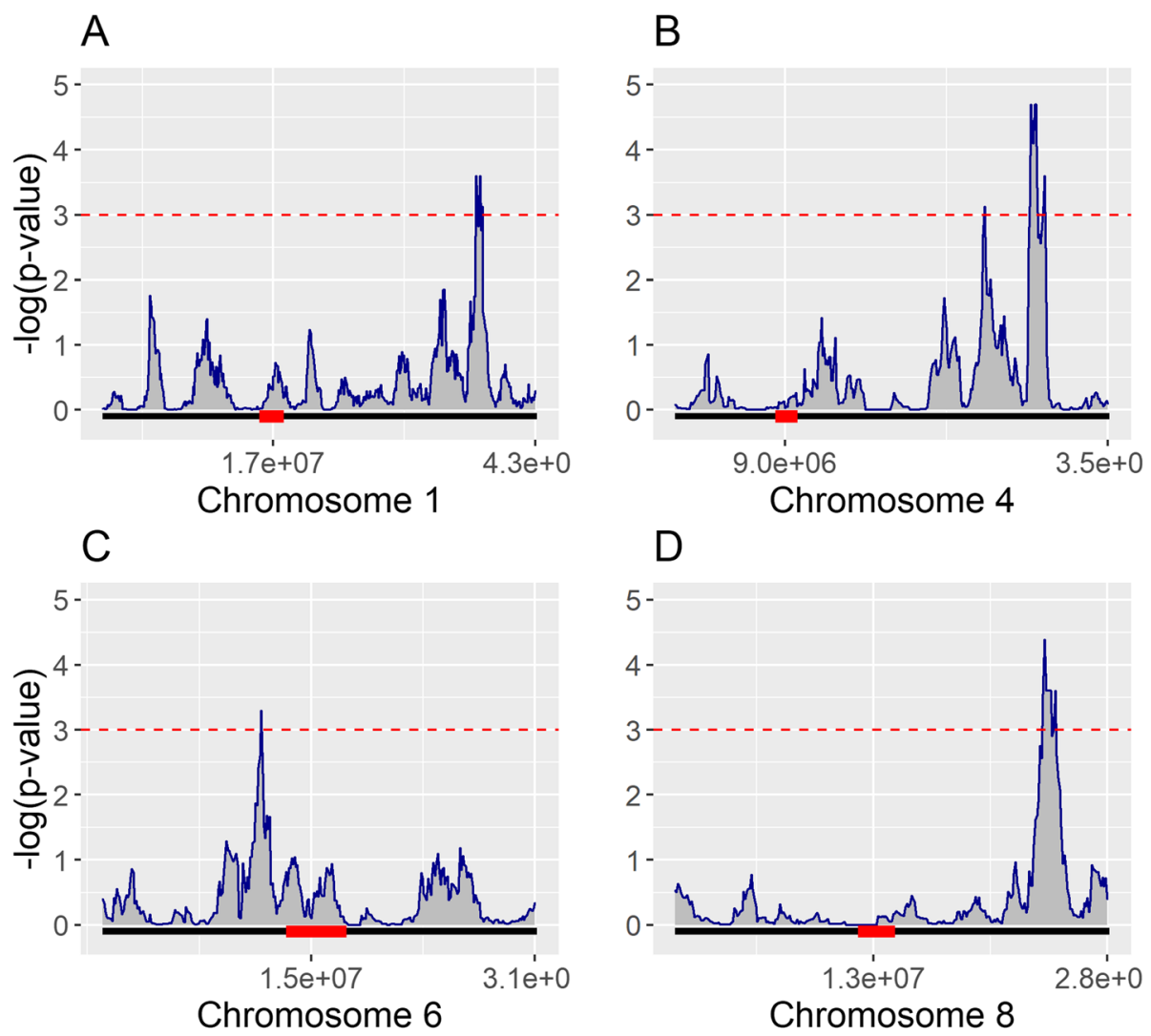

Window Size: 1500000

Fig. 5 Salt-specific Heritable Gene Enrichment. Plots A-D represent chromosomes 1, 4, 6, and 8 respectively. The black lines at the bottom of each plot represent the relative chromosome length, with the position and relative size of pericentromeric regions indicated by overlapping red boxes. Using a sliding window size of $1.5 \mathrm{Mb}$ at $100 \mathrm{~Kb}$ intervals, chromosomes were tested for enrichment of genes with salt-specific heritability using all genes with heritable expression (salt-specific, optimal-specific, and general) as the null distribution. P-values were adjusted for multiple-testing using a permutation based approach. Using a critical value of 0.001 , indicated by the dashed red line, significant windows enriched for saltspecific heritability were identified on chromosomes 1, 4, 6, and 8

missing values tend to have high TPM expression values. However, when a gene had a zero value, in any sample, then most non-zero values were in the LE distribution. Furthermore, when these patterns were compared between salt and control conditions, there were no genes that switched from repressed expression to constitutive expression in the population. Considering that four times as many genes shifted between mixed and repressed states (1939 genes) compared to genes that shifted between mixed and constitutive states (454 genes), one explanation is that many of these genes are located within chromosomal regions that are still largely repressed, but that this repression is incomplete and a low level of transcription still occurs. However, it is also possible that some of these conditional lowly expressed genes are being translated into proteins. Given that RNA-seq samples in this experiment consisted of homogenized shoot samples containing multiple cell types, cell-type specific expression could also explain genes that are lowly expressed. While the sample size $(n=336$ samples; 84 genotypes $\times 2$ conditions $\times 2$ biological replicates) was too small to reliably calculate the heritability

Table 2 Genome windows enriched for salt-specific heritable expression

\begin{tabular}{lllll}
\hline Chromosome & Start Position & End Position & Heritable genes & Fisher's test adjusted $\boldsymbol{p}$-value \\
\hline 1 & $36,450,000$ & $38,550,000$ & 19 & $2.5 \mathrm{E}-04$ \\
4 & $24,550,000$ & $26,050,000$ & 17 & $7.5 \mathrm{E}-04$ \\
4 & $28,250,000$ & $30,950,000$ & 19 & $2.0 \mathrm{E}-05$ \\
6 & $12,150,000$ & 13 & $5.0 \mathrm{E}-04$ \\
8 & $23,650,000$ & $25,650,000$ & 17 & $4.0 \mathrm{E}-05$ \\
\hline
\end{tabular}


of mixed and repressed gene expression using logistic models, PCA of the gene expression matrix encoded as ordinal zero, low, or high expression suggests that there is a large amount of additional transcriptional variance that closely matches the genotypic population structure (Additional File 1, Fig. S10). This variation may not be captured in current RNA-seq approaches that only consider TPMs from the HE distribution such as differential expression models using the negative-binomial distribution.

Regarding the notion that LE genes are not translated into proteins, this assumption is based on limited evidence that compared different cell types in different conditions. However, it may be too early to rule out potential translation of LE genes. Plant genomes have been shown to undergo drastic heterochromatic reorganization in response to abiotic stimuli including salt-stress [30]. The high correlation between LE genes and heterochromatic regions of the genome may suggest that rather than being untranslated, the low expression of these genes could be related to cell type or conditionspecific responses, which would lead to their proteins not being observed in previous proteomics studies that used different conditions and genotypes.

\section{Heritability}

The importance of using biological replicates for differential gene expression analysis has already been explored $[31,32]$ but this research also indicates that biological replicates provide important information for models estimating gene expression heritability. Considering the inherent noise that can be introduced by natural variation in gene expression such as circadian rhythm, the inclusion of biological replicates should be considered an indispensable aspect of RNA-seq experimental design. Previous research investigating the statistical power of RNA-seq based differential expression analysis indicated that at least six biological replicates were required to identify the majority of differentially expressed [32]. However, no studies have explored how increasing the number of biological replicates can improve the power of models that estimate gene expression heritability. Considering that these models can also benefit from increasing the number of genotypes, there is need for quantifying the power trade-off between the number of genotypes and the number of biological replicates for accurately estimating gene expression heritability.

Another result of interest is that the two-step GREML showed only moderate correlation with both the replicate-based and one-step GREML estimates. Differences in how genetic effects are distributed may explain this. Previous reports on eQTLs underlying gene expression heritability in humans suggest that highly heritable gene expression tends to be controlled by relatively few cis eQTLs with strong, non-additive, effects [33, 34]. Conversely, heritable complex traits and moderately heritable gene expression tend to be controlled by many small additive effect mutations $[35,36]$. This difference in how genetic effects are distributed may explain why GREML heritability estimates using mean expression was only lowly correlated with repeatability. Previous studies investigating heritability in human populations (with a much larger sample size than this study) split markers into separate cis and trans components in the GREML model where the cis random effects only included markers surrounding the gene being tested with the remaining markers included in the model as a separate trans random effect [13]. The approach for splitting cis and trans components in these studies used only markers within a $1 \mathrm{Mb}$ fixed window around a gene as the cis component (that was likely to capture any promoter regions) and treated all other markers as a separate trans component. The purpose for this is that mutations near the coding sequence and surrounding promoters seem more likely to have large effects on gene expression and thus would follow a different underlying distribution of effect sizes compared to mutations occurring elsewhere in the genome. In these human studies, the average overall mean heritabilities were reported to be between 0.15 and 0.26 with the proportion of heritability explained by cis markers ranging from 20 to $40 \%$ depending on the tissue and population studied. A smaller microarray-based eQTL study in an A. thaliana RIL population reported a similar heritability distribution [2]. Notably, they also observed many genes that exhibited transgressive segregation and suggested that nonadditive genetic variation may be significantly contributing to overall expression heritability in plants.

The sample size of the data used in this study was too low to reasonably split markers into separate cis and trans random effects in the additive GREML model to allow for direct comparison to previous studies. However, the low correlation between the two-step GREML additive-only model and the one-step GREML model that included replicates as a random effect supports the idea that gene expression traits have a genomic architecture that cannot be captured well by treating all genome-wide markers as a single additive random effect distribution. One possible alternative for modeling gene expression traits that could avoid an arbitrary fixed window for splitting markers into cis and trans components is to use variable selection methods that can accommodate mixed distributions of marker effects. There is considerable similarity between the previously used strategy of modeling separate cis and trans components and Bayesian models used for genomic selection which can accommodate many different prior distribution assumptions [37]. However, challenges remain for testing 
whether these Bayesian methods can more effectively estimate marker effects underlying transcriptome-wide gene expression. First, there are many different prior distributions proposed for performing Bayesian genomic selection and selecting a suitable prior distribution is nontrivial considering that the underlying architectures of heritable gene expression are heterogeneous [38, 39]. Secondly, even with parallelization, the Markov chain Monte Carlo algorithms involved have considerably higher computational costs compared to GREML making intensive testing difficult.

\section{Chromosomal structure and conformation}

The strong correlation between gene expression chromosome densities and $\mathrm{HiC}$ compartment predictions supports the paradigm that pericentromeric regions play an important transcriptional regulatory role in the 3D conformation of chromosomes in the nucleus and primarily correspond to heterochromatic B compartments in rice. For example, HE genes with constitutive expression patterns are more likely to be located in euchromatic A compartments, while LE genes with low and repressed expression are more likely to be located in heterochromatic B compartments. Therefore, the strong relationship identified between a gene's expression pattern and its position in the chromosome may have important implications for predicting the effects of structural variations such as translocation or gene duplication events. Such an understanding may improve studies exploring the role of duplicated genes, as it may be essential to consider where in the chromosome duplicate genes are located and how the surrounding regulatory landscape is different (such as a shift in chromatin compartment).

\section{Overlap between salt stress QTLs and expression heritability}

An interesting observation regarding the overlap between salt-tolerance associated QTLs identified in the RDP1 population using GWAS and the windows enriched for salt-stress specific heritable expression is that the current putative causal gene underlying the largest salt-tolerance QTL in this population, OsHKT1;1 (LOC_Os04g51820), did not exhibit heritable gene expression after accounting for population structure. However, many genes within close proximity to this gene did have heritable expression and this region was particularly enriched for salt-specific expression heritability. This indicates that causal genes underlying complex phenotypes may have indirect effects on gene networks. One possible explanation for this is that genes that coparticipate in shared biological pathways have been shown to cluster in the same chromosomal region [40]. However, this clustering does not occur in all plant pathways and there are currently many theories for why some pathways are genomically clustered and others are not [41]. One of these theories is the 'coinheritance argument' where genetic linkage of genes with shared roles in a complex trait can promote the accumulation of favorable genes and reduce risk of disruption via recombination. Given that salt-tolerance is a trait in rice with a history of both evolutionary and artificial selection, this theory may explain the clustering observed.

\section{Implications}

Results show that the relatively small sample sizes in this study (compared to typical GWAS studies) were able to identify regions of the genome enriched for conditionspecific heritable gene expression. This approach could be used to identify genes involved with conditional transcriptomic plasticity. Identifying heritable genes with genotype-by-environment specific behaviors may be useful to breeders in MAS approaches to select for mutations with more isolated trait-specific effects, across genotypes, and avoid the selection of mutations with strong epistatic effects.

While it is generally accepted that the genome-wide distribution of marker effects for complex traits is nonuniform, there are few approaches for determining how non-uniformity relates to the physical genome. However, the chromosome-level patterns of gene expression heritability observed in this study could potentially be used as prior estimates of possible marker effect distributions for Bayesian genomic selection models. Even if the underlying true distribution may have cryptic conditionspecific components outside the scope of available RNAseq data, a large proportion of heritable expression was observed for both conditions. For example, there were multiple regions of the genome with relatively few genes with heritable gene expression for either condition. Markers within these regions could be assigned low prior probabilities of having strong effects. In contrast, we also identified regions of the genome with high general and condition-specific heritable expression. Markers within this region could be assigned higher prior weights, especially when they are located in trait related conditional hotspots.

\section{Future considerations}

The increasing number of studies in plants utilizing standardized genetic diversity panels for producing multi-omics based data is allowing for rich multidimensional research into biological systems. The results observed in this study provide a valuable initial point of comparison. While further experiments investigating these hotspots enriched for salt-specific heritable expression are required for validation, results regarding missing values and their relation to bimodal expression patterns 
highlight the need for more overlapping -omics data. First, use of larger genotype panels for transcriptomic sequencing with more biological replications would improve the precision of heritability estimates, allow for finer cross-conditional comparisons, and allow for more powerful transcriptome-wide exploration of trans genetic effects on gene expression. Second, access to highresolution chromatin contact maps would allow for further investigation into the roles that lower-level chromatin structures (such as topologically association domains) play in regulatory variation for how plants respond to stress. While many RNA-seq experiments primarily focus on analyzing highly expressed genes, this research indicates that genes with low non-zero expression also have distinct spatial patterns that may provide evolutionary value and should be further explored. Furthermore, the addition of conditionally matched proteomics data would help resolve the open question if any of these lowly-expressed genes are ever translated into proteins.

\section{Conclusions}

Transcriptional regulation is considered to be a major mechanism for how plants respond to environmental changes. and developing a better understanding of genetic variation in stress-induced gene expression may lead to improved methods for crop breeding. This research sought to explore patterns of condition-specific heritable gene expression across a genetically diverse population and discovered a bimodal pattern of highly and lowly expressed genes that was highly correlated with chromosome-wide A/B chromatin compartments and was mostly stable across both genotypes and conditions. However, we also discovered a contrasting pattern of region-specific hotspots that were significantly enriched with genes that have heritable expression only during stress conditions. Together, these findings suggest that genetic variation in rice does not likely have large effects on high-level chromatin structures such as A/B compartments, but there may be smaller regional effects on lower-level chromatin structures that can lead to neighborhoods of genes with shared heritable variations in gene expression.

\section{Methods}

\section{Genotype data}

All rice accessions used in this research are from the Rice Diversity Panel 1. This panel consists of 421 purified, homozygous rice accessions that include both landraces and elite rice cultivars worldwide. Genotypes for the entire panel were obtained from the online project repository for the Rice Diversity Project [42]. In particular, this research used a set of $44 \mathrm{k}$ SNPs obtained from a combination of array and sequencing-based approaches. Missing genotypes were imputed using LDkNNi [43]. The cross-validated accuracy using known genotypes was found to be highly accurate $\left(R^{2}=0.98\right)$. Markers with an imputed minor allele frequency of less than $5 \%$ were removed leaving a total of 31,374 markers for further analysis.

\section{Gene expression data}

RNA-seq sequence files for a subset of rice accessions $(n=92)$ from the RDP1 panel were identified and sourced from the National Center for Biotechnology Information sequence read archive (SRA) listed under Gene Expression Omnibus (GEO) project GSE98455. This previously published data originates from a project investigating salt-stress related gene co-expression network modules [28]. Briefly, seedlings of each accession were subjected to either optimal or salt-stress conditions for $24 \mathrm{~h}$ and afterwards, shoot-tissue RNA was extracted and sequenced. Each treatment has two biological replicates originating from separate but genetically identical inbred accessions for a total of 368 RNA-seq samples. Only accessions that had replicates for both conditions were used $(n=84)$ (Additional File 2, Table S4) for a filtered total of 336 samples.

RNA-seq files were downloaded and processed using the GEMmaker v1.1 pipeline for gene expression analysis [44]. This pipeline streamlines the process of calculating a gene expression matrix (GEM) from large numbers of raw FASTQ [45] sequencing files. GEMmaker was configured to download the GEO project GSE98455 sequence files using the SRA toolkit [46], perform quality control with FastQC [47] and quantify Transcripts-per-million (TPM) [48] expression values using Kallisto [49], a pseudo-alignment based tool. Gene annotations from the Michigan State University Rice Genome Annotation Project (MSU release 7) were used for pseudo-alignment, which are based on the International Rice Genome Sequencing Project reference genome (Os-Nipponbare-Reference-IRGSP-1.0) [50]. TPM values were calculated at the gene level rather than the isoform level due to limited annotation of alternative splicing in rice. TPM values were $\log 2$ transformed. The sample and gene-wise distributions of mean $\log 2$ TPM and proportion of missing values were assessed.

\section{Structural analysis}

Prior research on this population's structure indicated that the panel has five major sub-groups [42]. We replicated the structural analysis with the subset of RDP1 individuals used in this study and found the same conclusion. Based on principal-component analysis (PCA), the top three components were found to capture a majority of genetic variance across subgroups (61\%) (Additional File 1, Fig. S11). Initial inspection of pairwise 
TPMs indicated the presence of population structure matching the major classes of rice identified from marker-based principal component analysis. Because of the high collinearity between markers separating these groups and clusters of gene expression, not accounting for this population structure could lead to inflated heritability estimates [51]. For linear mixed models, this can be addressed by including subpopulation identifiers or principal components as fixed effects within the model. However, for simpler repeatability metrics, such as Spearman correlation, this is not possible. Therefore, expression values were adjusted to remove the major subpopulation effects prior to calculating any heritability estimates. This was done by fitting the expression values for each gene with a linear model including the top three principal components calculated from the genotype matrix as independent fixed effects. The remaining residuals were then used as adjusted gene expression values for calculating heritabilities for each gene. This adjustment was done separately for each condition and replicate. The distribution of the impact of structural adjustment indicates that while the repeatability of most genes slightly decreased when structure was removed, this decrease was larger for genes with clear clustering related to the population structure (Additional File 1, Fig. S12).

\section{Heritability calculations}

All statistics were performed using $\mathrm{R}$ 3.6.0 [52]. Condition-specific heritabilities were calculated for the expression of each gene and condition using multiple methods (Additional File 2, Tables S5 and S6). Genes with $<5 \%$ missing values across all samples were used in heritability analysis. Heritability was first estimated using the similarity of expression between biological replicates. Because all accessions were inbred lines, and conditions were tightly controlled between lines, the Pearson or Spearman correlations between replicates within a shared condition were calculated as an upper-bound of the heritability of gene expression for that condition. Heritability was also calculated using single-step and two-step GREML algorithms implemented in the R 'heritability' package version 1.3 [53]. These GREML methods use a GRM calculated from genotypes to solve a linear mixed model for a quantitative phenotype with the efficient mixed-model association algorithm commonly used for estimating heritability and genomic selection of agronomic phenotypes in crops [54]. The GRM was estimated with the genetic ridge-regression $\mathrm{R}$ package 'rrBLUP' using version 4.6.1 [55]. Because expression values were already adjusted for subpopulation structure, principal components were not included in the GREML models. Default values were used for the convergence criterion (eps) and maximum iterations (max.iter). Two-step GREML calculation was performed by first calculating the genotypic expression mean across replicates and then regressing these mean values with a linear model treating kinship as a random effect using the marker_h2_means function. Single-step GREML was estimated using the marker_h2 function but instead of regressing the genotypic mean, it includes replicate variance in the model as an added random effect [53]. The significance of heritability estimates compared to randomized gene expression were measured based on a one-tailed shuffled permutation test where the expression values for each gene were randomly shuffled for 40 , 000 iterations. This number of iterations was chosen based on hardware capabilities within a 48-h window. Assuming that randomly shuffled gene expression vectors should not be heritable, the resulting heritability distribution of randomized expression was used to calculate a significance threshold based on a fixed type-1 error rate $(\square=0.01)$. This threshold was then used to test whether each gene was significantly heritable (Fig. 3a). Genes were then classified if they were significantly heritable under control, salt-stress, or both conditions (Fig. 3b).

\section{Spatial enrichment analysis}

The spatial distribution of expressed genes and their calculated heritabilities across each chromosome were compared between the control and stress conditions. This was done using a sliding window $(1.5 \mathrm{Mb})$ and sampling interval to calculate the frequency or density of genes across each chromosome. Enrichment of saltspecific heritable genes for each window was determined using a one-tailed Fisher's exact test. Test $p$-values were adjusted for multiple testing bias by calculating a nulldistribution for each window. First, random gene subsets of equivalent size to the number of salt-specific heritable genes were randomly drawn from all heritable genes. For each random subset, a Fisher's test was performed for each window resulting in a window-specific $p$-value. This process was bootstrapped for 4000 iterations $(n=$ 4000 and the resulting $p$-value distributions were used to calculate adjusted $p$-value quantiles. For windows where 4000 iterations were not enough to assign a $p$ value quantile, these windows were further tested for up to 50,000 iterations until a stable quantile estimate was obtained. Genes significantly enriched for salt-specific heritable expression within windows were tested for functional term enrichment using the Comprehensive Annotation of Rice Multi-Omicstool [56].

\section{Multi-omics integration}

The spatial distribution of gene expression heritability was then overlaid with other types of -omics data. The densities of genes with different expression patterns 
(constitutive, mixed and repressed) were tested for correlation with chromatin $\mathrm{A} / \mathrm{B}$ compartment eigenvectors from previously published $\mathrm{Hi}-\mathrm{C}$ analysis [27]. The $\mathrm{Hi}-\mathrm{C}$ analysis used a fixed bin width of $500 \mathrm{~kb}$. This fixed window size was then used to calculate the gene densities of different expression patterns and correlated to the $\mathrm{A} / \mathrm{B}$ eigenvectors.

\section{Abbreviations}

eQTL: Expression quantitative trait locus; GEM: Gene Expression Matrix; GEO: Gene Expression Omnibus; GREML: Genomic relatedness restricted maximum likelihood; GRM: Genetic relationship matrix; GWAS: Genome-wide association study; HE: Highly expressed; Hi-C: High-throughput chromatin conformation capture; LE: Lowly expressed; PCA: Principal component analysis; SNP: Single-nucleotide polymorphism; SRA: Sequence read archive; TPM: Transcripts per million

\section{Supplementary Information}

The online version contains supplementary material available at https:/doi. org/10.1186/s12863-021-00970-7.

\section{Additional file 1. \\ Additional file 2}

\section{Acknowledgments}

We thank Lei Gong and staff at the Key Laboratory of Molecular Epigenetics of the Ministry of Education at Northeast Normal University, Changchun, China for providing their $\mathrm{Hi}-\mathrm{C} \mathrm{A} / \mathrm{B}$ compartment eigenvalue data.

\section{Authors' contributions}

M.M. conceived, designed, and executed the experimental approach. S.F. assisted with the computational processing of data. Z.Z. and S.F. provided guidance in the interpretation of results. M.M. drafted the manuscript that was subsequently edited by S.F. and Z.Z. All authors have read and approved the manuscript.

\section{Funding}

This work was supported by the USDA National Institute of Food and Agriculture (Hatch project 1014919, Award \#s 2018-70005-28792, 201967013-29171, and 2020-67021-32460), and the Washington Grain Commission (Endowment and Award \#s 126593 and 134574). These funding agencies played no role in the design of the study, data collection, analysis and interpretation, or in writing the manuscript.

\section{Availability of data and materials}

All RNA-seq data is publicly available via the Gene Expression Omnibus under project GSE98455 [28]. Genotypes for all 84 rice accessions (Additional File 2, Table S4) were obtained from the online project repository for the Rice Diversity Project [42]. A repository containing processed RNA-seq TPM counts, filtered genotypes, Hi-C A/B component data, and R scripts for replicating heritability analysis and visualization of results are available as a public repository on the Open Science Framework [57]

\section{Declarations}

Ethics approval and consent to participate

Not applicable.

\section{Consent for publication}

Not applicable.

\section{Competing interests}

The authors declare they have no competing interests.

\section{Author details}

'Molecular Plant Sciences Program, Washington State University, French Ad 324G, Pullman, WA 99164, USA. ²Department of Crops and Soils, Washington
State University, 105 Johnson Hall, Pullman, WA 99164, USA. ${ }^{3}$ Department of Horticulture, Washington State University, 149 Johnson Hall, Pullman, WA 99164, USA.

Received: 11 January 2021 Accepted: 6 May 2021

Published online: 27 May 2021

\section{References}

1. Hardy J, Singleton A. Genomewide association studies and human disease. N Engl J Med. 2009;360(17):1759-68. https://doi.org/10.1056/NEJMra 0808700.

2. West MAL, Kim K, Kliebenstein DJ, Van Leeuwen H, Michelmore RW, Doerge RW, et al. Global eQTL mapping reveals the complex genetic architecture of transcript-level variation in Arabidopsis. Genetics. 2007;175(3):1441-50. https://doi.org/10.1534/genetics.106.064972.

3. Liu H, Luo X, Niu L, Xiao Y, Chen L, Liu J, et al. Distant eQTLs and noncoding sequences play critical roles in regulating gene expression and quantitative trait variation in maize. Mol Plant. 2017;10(3):414-26. https://doi org/10.1016/j.molp.2016.06.016

4. Ingvarsson PK, Street NR. Association genetics of complex traits in plants. New Phytol. 2011;189(4):909-22. https://doi.org/10.1111/j.1469-8137.2010.03 593.x.

5. Hammond JP, Mayes S, Bowen HC, Graham NS, Hayden RM, Love CG, et al. Regulatory hotspots are associated with plant gene expression under varying soil phosphorus supply in brassica rapa. Plant Physiol. 2011;156(3): 1230-41. https://doi.org/10.1104/pp.111.175612.

6. Visscher PM, Hill WG, Wray NR. Heritability in the genomics era - concepts and misconceptions. Nat Rev Genet. 2008;9(4):255-66. https://doi.org/10.103 8/nrg2322.

7. Piepho HP, Möhring J. Computing heritability and selection response from unbalanced plant breeding trials. Genetics. 2007;177(3):1881-8. https://doi org/10.1534/genetics.107.074229

8. Tenesa A, Haley CS. The heritability of human disease: estimation, uses and abuses. Nat Rev Genet. 2013;14(2):139-49. https://doi.org/10.1038/nrg3377.

9. Yang J, Lee SH, Goddard ME, Visscher PM. GCTA: a tool for genome-wide complex trait analysis. Am J Hum Genet. 2011;88(1):76-82. https://doi.org/1 0.1016/j.ajhg.2010.11.011.

10. Hyun MK, Zaitlen NA, Wade CM, Kirby A, Heckerman D, Daly MJ, et al. Efficient control of population structure in model organism association mapping. Genetics. 2008;178(3):1709-23. https://doi.org/10.1534/genetics.1 07.080101.

11. Endelman JB. Ridge regression and other kernels for genomic selection with R package rrBLUP. Plant Genome. 2011;4(3):250-5. https://doi.org/10.3 835/plantgenome2011.08.0024.

12. Wright FA, Sullivan PF, Brooks Al, Zou F, Sun W, Xia K, et al. Heritability and genomics of gene expression in peripheral blood. Nat Genet. 2014;46(5): 430-7. https://doi.org/10.1038/ng.2951.

13. Lloyd-Jones LR, Holloway A, McRae A, Yang J, Small K, Zhao J, et al. The genetic architecture of gene expression in peripheral blood. Am J Hum Genet. 2017:100(2):228-37. https://doi.org/10.1016/j.ajhg.2016.12.008.

14. Mohanta TK, Bashir T, Hashem A, Abd Allah EF. Systems biology approach in plant abiotic stresses. Plant Physiol Biochem. 2017;121:58-73. https://doi. org/10.1016/j.plaphy.2017.10.019

15. Mar JC. The rise of the distributions: why non-normality is important for understanding the transcriptome and beyond. Biophys Rev. 2019;11(1):8994. https://doi.org/10.1007/s12551-018-0494-4

16. Hart T, Komori HK, LaMere S, Podshivalova K, Salomon DR. Finding the active genes in deep RNA-seq gene expression studies. BMC Genomics. 2013;14(1):1-7. https://doi.org/10.1186/1471-2164-14-778.

17. Hebenstreit D, Fang M, Gu M, Charoensawan V, Van Oudenaarden A, Teichmann SA. RNA sequencing reveals two major classes of gene expression levels in metazoan cells. Mol Syst Biol. 2011;7(1):497. https://doi. org/10.1038/msb.2011.28.

18. Allshire RC, Madhani HD. Ten principles of heterochromatin formation and function. Nat Rev Mol Cell Biol. 2018;19(4):229-44. https://doi.org/10.1038/ nrm.2017.119.

19. She W, Grimanelli D, Rutowicz K, Whitehead MWJ, Puzio M, Kotliński M, et al. Chromatin reprogramming during the somatic-to-reproductive cell fate transition in plants. Dev. 2013;140(19):4008-19. https://doi.org/10.1242/ dev.095034. 
20. Rosa S, Ntoukakis V, Ohmido N, Pendle A, Abranches R, Shaw P. Cell differentiation and development in Arabidopsis are associated with changes in histone dynamics at the single-cell level. Plant Cell. 2014;26(12):4821-33. https://doi.org/10.1105/tpc.114.133793.

21. Asensi-Fabado MA, Amtmann A, Perrella G. Plant responses to abiotic stress: the chromatin context of transcriptional regulation. Biochimica et Biophysica Acta - Gene Regulatory Mechanisms. 1860;2017:106-22.

22. Kim JM, To TK, Nishioka T, Seki M. Chromatin regulation functions in plant abiotic stress responses. Plant Cell Environ. 2010;33(4):604-11. https://doi. org/10.1111/j.1365-3040.2009.02076.x.

23. Tittel-Elmer M, Bucher E, Broger L, Mathieu O, Paszkowski J, Vaillant I. Stressinduced activation of heterochromatic transcription. PLOS Genet. 2010;6(10): e1001175. https://doi.org/10.1371/journal.pgen.1001175.

24. Okamoto $\mathrm{H}$, Hirochika $\mathrm{H}$. Silencing of transposable elements in plants. Trends Plant Sci. 2001;6(11):527-34. https://doi.org/10.1016/S1360-1385(01 02105-7.

25. Lippman Z, Gendrel AV, Black M, Vaughn MW, Dedhia N, McCombie WR, et al. Role of transposable elements in heterochromatin and epigenetic control. Nature. 2004;430(6998):471-6. https://doi.org/10.1038/nature02651.

26. Dong P, Tu X, Li H, Zhang J, Grierson D, Li P, et al. Tissue-specific hi-C analyses of rice, foxtail millet and maize suggest non-canonical function of plant chromatin domains. J Integr Plant Biol. 2020;62(2):201-17. https://doi. org/10.1111/jipb.12809.

27. Dong Q, Li N, Li X, Yuan Z, Xie D, Wang X, et al. Genome-wide hi-C analysis reveals extensive hierarchical chromatin interactions in rice. Plant J. 2018; 94(6):1141-56. https://doi.org/10.1111/tpj.13925.

28. Campbell MT, Bandillo N, Razzaq F, Al Shiblawi A, Sharma S, Liu K, et al. Allelic variants of OsHKT1;1 underlie the divergence between indica and japonica subspecies of rice (Oryza sativa) for root sodium content. PLoS Genet. 2017;13(6):e1006823. https://doi.org/10.1371/journal.pgen.1006823.

29. Kawahara Y, de la Bastide M, Hamilton JP, Kanamori H, Mccombie WR, Ouyang $\mathrm{S}$, et al. Improvement of the oryza sativa nipponbare reference genome using next generation sequence and optical map data. Rice. 2013; 6(1):3-10. https://doi.org/10.1186/1939-8433-6-4

30. Probst AV, Mittelsten SO. Stress-induced structural changes in plant chromatin. Curr Opin Plant Biol. 2015;27:8-16. https://doi.org/10.1016/j.pbi.2 015.05.011.

31. Liu Y, Zhou J, White KP. RNA-seg differential expression studies: more sequence or more replication? Bioinformatics. 2014;30(3):301-4. https://doi. org/10.1093/bioinformatics/btt688.

32. Schurch NJ, Schofield P, Gierliński M, Cole C, Sherstnev A, Singh V, et al. How many biological replicates are needed in an RNA-seq experiment and which differential expression tool should you use? RNA. 2016;22(6):839-51. https://doi.org/10.1261/rna.053959.115.

33. Gilad Y, Rifkin SA, Pritchard JK. Revealing the architecture of gene regulation: the promise of eQTL studies. Trends Genet. 2008;24(8):408-15. https://doi.org/10.1016/j.tig.2008.06.001.

34. Wheeler HE, Shah KP, Brenner J, Garcia T, Aquino-Michaels K, Cox NJ, et al. Survey of the heritability and sparse architecture of gene expression traits across human tissues. PLoS Genet. 2016;12(11):e1006423. https://doi.org/1 0.1371/journal.pgen.1006423.

35. Albert FW, Kruglyak $L$. The role of regulatory variation in complex traits and disease. Nat Publ Gr. 2015;16(4):197-212. https://doi.org/10.1038/nrg3891.

36. Holland JB. Genetic architecture of complex traits in plants. Curr Opin Plant Biol. 2007;10(2):156-61. https://doi.org/10.1016/j.pbi.2007.01.003.

37. Habier D, Fernando RL, Kizilkaya K, Garrick DJ. Extension of the bayesian alphabet for genomic selection. BMC Bioinformatics. 2011;12(1):186. https:// doi.org/10.1186/1471-2105-12-186.

38. Gianola D. Priors in whole-genome regression: the Bayesian alphabet returns. Genetics. 2013;194(3):573-96. https://doi.org/10.1534/genetics.113.1 51753.

39. Kärkkäinen HP, Sillanpää MJ. Back to basics for Bayesian model building in genomic selection. Genetics. 2012;191(3):969-87. https://doi.org/10.1534/ genetics.112.139014

40. Field B, Osbourn A. Order in the playground. Mob Genet Elements. 2012; 2(1):46-50. https://doi.org/10.4161/mge.19348.

41. Nützmann HW, Huang A, Osbourn A. Plant metabolic clusters - from genetics to genomics. New Phytol. 2016;211(3):771-89. https://doi.org/1 0.1111/nph.13981.

42. Zhao K, Tung CW, Eizenga GC, Wright MH, Ali ML, Price AH, et al. Genomewide association mapping reveals a rich genetic architecture of complex traits in Oryza sativa. Nat Commun. 2011;2(1):1-10. https://doi.org/10.1038/ ncomms1467.

43. Money D, Gardner K, Migicovsky Z, Schwaninger H, Zhong GY, Myles S. Linklmpute: fast and accurate genotype imputation for nonmodel organisms. G3 genes, genomes. Genet. 2015;5(11):2383-90. https://doi.org/1 $0.1534 / \mathrm{g} 3.115 .021667$.

44. Hadish J. GEMmaker. https://github.com/SystemsGenetics/GEMmaker. Accessed 16 Nov 2020.

45. Cock PJA, Fields CJ, Goto N, Heuer ML, Rice PM. The sanger FASTQ file format for sequences with quality scores, and the Solexa/Illumina FASTQ variants. Nucleic Acids Res. 2009;38(6):1767-71. https://doi.org/10.1093/nar/ gkp1137.

46. Sherry S, Xiao C, Durbrow K, Kimelman M, Rodarmer K, Shumway M, et al. Ncbi sra toolkit technology for next generation sequence data. In: Plant and Animal Genome XX Conference; 2012. http://1000gconference.sph.umich. edu/abstracts/62ac2670d47b50dc8bd31cfad96c52db.pdf. Accessed 16 Nov 2020.

47. Andrews S. FastQC: a quality control tool for high throughput sequence data. 2010. https://www.bioinformatics.babraham.ac.uk/projects/fastqc/. Accessed 16 Nov 2020.

48. Wagner GP, Kin K, Lynch VJ. Measurement of mRNA abundance using RNAseq data: RPKM measure is inconsistent among samples. Theory Biosci. 2012;131(4):281-5. https://doi.org/10.1007/s12064-012-0162-3.

49. Bray NL, Pimentel H, Melsted P, Pachter L. Near-optimal probabilistic RNAseq quantification. Nat Biotechnol. 2016;34(5):525-7. https://doi.org/10.1038/ nbt.3519.

50. Mizuno H, Kawahara Y, Wu J, Katayose $Y$, Kanamori H, Ikawa H, et al. Asymmetric distribution of gene expression in the centromeric region of rice chromosome 5. Front Plant Sci. 2011;2:16

51. Browning SR, Browning BL. Population structure can inflate SNP-based heritability estimates supplemental data. Am J Hum Genet. 2011;89(1):1913. https://doi.org/10.1016/j.ajhg.2011.05.025.

52. R Core Team. R: A Language and environment for statistical computing. Vienna, Austria: R Foundation for Statistical Computing; 2020.

53. Kruijer W, Boer MP, Malosetti M, Flood PJ, Engel B, Kooke R, et al. Markerbased estimation of heritability in immortal populations. Genetics. 2014;199: 379-98.

54. Zhu H, Zhou X. Statistical methods for SNP heritability estimation and partition: a review. Comput Struct Biotechnol J. 2020;18:1557-68. https://doi. org/10.1016/j.csbj.2020.06.011.

55. Endelman JB, Jannink JL. Shrinkage estimation of the realized relationship matrix. G3 genes, genomes. Genet. 2012;2:1405-13.

56. Wang J, Qi M, Liu J, Zhang Y. CARMO: a comprehensive annotation platform for functional exploration of rice multi-omics data. Plant J. 2015; 83(2):359-74. https://doi.org/10.1111/tpj.12894

57. McGowan M. Rice_RDP1_salt_stress; 2021. https://osf.io/fd9sc/. https://doi. org/10.17605/OSF.IO/FD9SC/.

\section{Publisher's Note}

Springer Nature remains neutral with regard to jurisdictional claims in published maps and institutional affiliations.

Ready to submit your research? Choose BMC and benefit from:

- fast, convenient online submission

- thorough peer review by experienced researchers in your field

- rapid publication on acceptance

- support for research data, including large and complex data types

- gold Open Access which fosters wider collaboration and increased citations

- maximum visibility for your research: over $100 \mathrm{M}$ website views per year

At $\mathrm{BMC}$, research is always in progress.

Learn more biomedcentral.com/submissions 